\title{
Commissioning a Vibrating Wire System for Quadrupole Fiducialization
}

\author{
Michael Y. Levashov, Zachary Wolf
}

September 5, 2007

\begin{abstract}
A vibrating wire system was constructed to fiducialize the quadrupoles between undulator segments in the LCLS. This note is a continuation of previous work to study the ability of the system to fulfill the fiducialization requirements.
\end{abstract}

\section{Introduction $^{1}$}

Quadrupoles will be placed between the undulator segments in LCLS to keep the electron beam focused as it passes through. The quadrupoles will be assembled with their respective undulator segments prior to being placed into the tunnel. Beam alignment will be used to center the quadrupoles, along with the corresponding undulators, on the beam. If there is any displacement between the undulator and the quadrupole axes in the assemblies, the beam will deviate from the undulator axis. If it deviates by more than $80 \mu m$ in vertical or $140 \mu \mathrm{m}$ in horizontal directions, the undulator will not perform as required by LCLS [1]. This error is divided between three sources: undulator axis fiducialization, quadrupole magnetic axis fiducialization, and assembly of the two parts. In particular, it was calculated that the quadrupole needs to be fiducialized to within $25 \mu \mathrm{m}$ in both vertical and horizontal directions.

A previous study [2] suggested using a vibrating wire system for finding the magnetic axis of the quadrupoles. The study showed that the method

\footnotetext{
${ }^{1}$ Work supported in part by the DOE Contract DE-AC02-76SF00515. This work was performed in support of the LCLS project at SLAC.
} 
has high sensitivity (up to $1 \mu m$ ) and laid out guidelines for constructing such a system.

There are 3 steps in fiducializing the quadrupole with the vibrating wire system. They are positioning the wire at the magnet center (step 1), finding the wire with position detectors (step 2), and finding the quadrupole tooling ball positions relative to the position detector tooling balls (step 3). [3]

A previous study ([4]) investigated the error associated with each step by using a permanent quadrupole magnet on an optical mover system. The study reported an error of $11 \mu \mathrm{m}$ for step 1 and a repeatability of $4 \mu \mathrm{m}$ for step 2. However, the set up used a FARO arm to measure tooling balls and didn't allow to accurately check step 2 for errors; an uncertainty of $100 \mu \mathrm{m}$ was reported. Therefore, even though the repeatability was good, there was no way to check that the error in step 2 was small.

Following the recommendations of that study, we used a CMM (Coordinate Measuring Machine) instead of the FARO arm for measuring the tooling balls. In addition, a roller cam positioner system ([5]) replaced the optical movers for moving the quadrupole. With the exception of the quadrupole itself, the system was identical to what will be used in fiducializing the undulator quadrupoles.

In this study, we investigate the new vibrating wire set up, including the error associated with each step of fiducialization.

\section{Setup}

For this study we used a setup similar to the previous vibrating wire system $[4]$.

Figure 1 shows most of the hardware components mounted on an optical table. Figure 2 shows most of the electronics used in the testing.

Although invisible in the pictures, a $100 \mu \mathrm{m}$ diameter wire is stretched between two sets of crossed pins (P1 and P2 in the figures). The ends of the wire are connected to a function generator $(\mathbf{F G})$. A weight $(\mathbf{W})$ keeps the wire under a constant tension. The wire goes through a quadrupole magnet (QM) that is on top of a supporting stand (ST). Note that instead of the quadrupoles that will be used in LCLS, a permanent quadrupole is used, since the electromagnets are not yet available. This, however, should have no effect on the validity of this study, because the field gradients in both magnets are similar. The stand contains a 5 degree of freedom cam positioner system, 


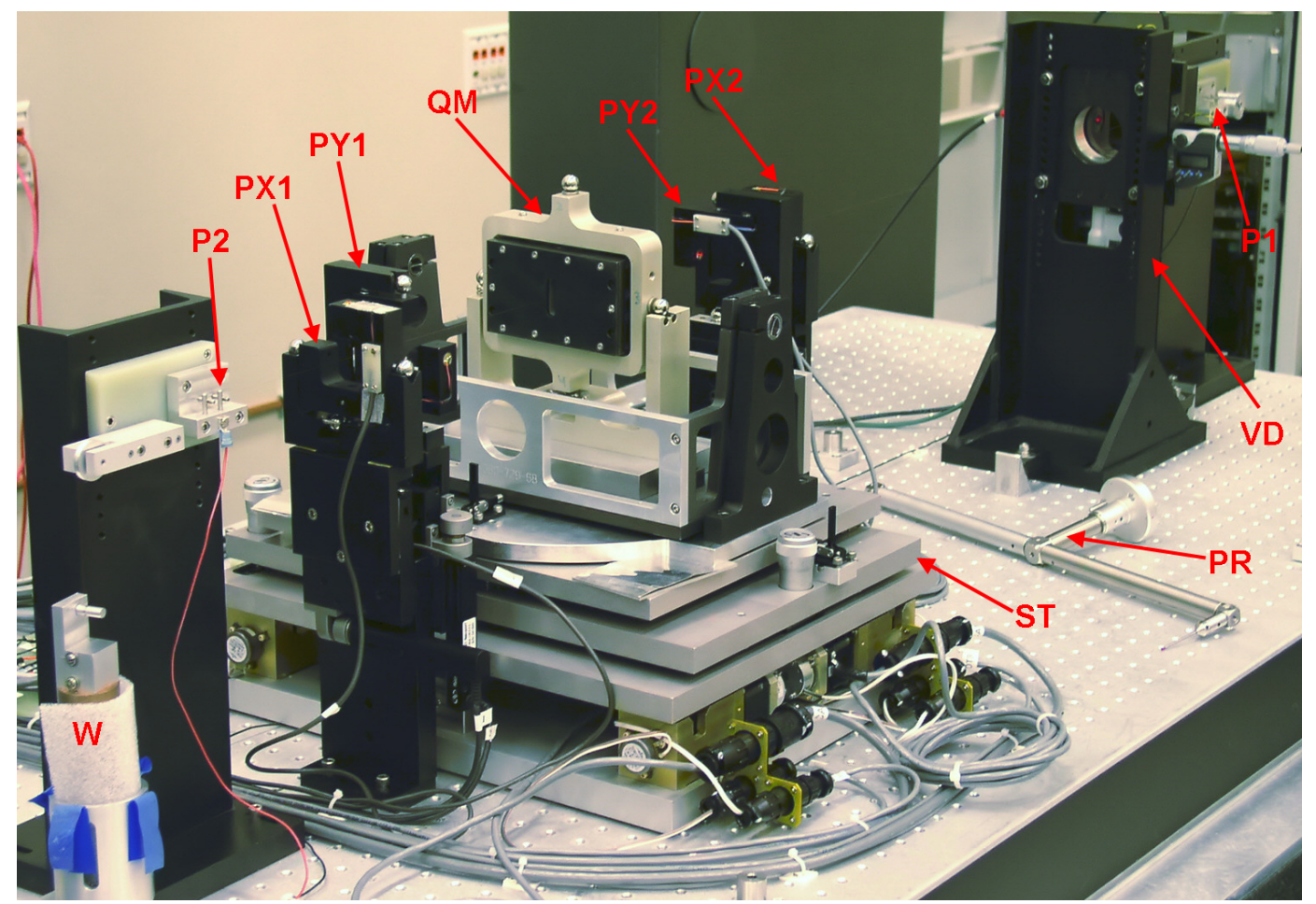

Figure 1: Setup

that allows it to translate in $x$ and $y$ and change roll, pitch, and yaw.

The wire position is measured by the $x$ (PX1 and PX2) and $y$ (PY1 and PY2) position sensors. The signals are amplified (PA1, PA2, PA3 and PA4) and fed into a voltmeter (VM1).

A laser sensor (VD) measures the $x$ and $y$ components of wire vibration. The signals from the sensor are amplified (VAX and VAY) and fed into lockin amplifiers (LIX and LIY), which are locked on to the function generator signal. At resonance frequency, the wire vibration is $90^{\circ}$ out of phase with the generator signal. This allows the relevant signal to be easily picked out from the noise by the lock-ins.

A second voltmeter (VM2) is used to read the position of each cam using potentiometers. It also contains a set of switches to release the brakes on the cam positioners before the start of each movement.

The lasers on all detectors, the potentiometers, and the brakes are powered by a low-noise power supply (PWS). 


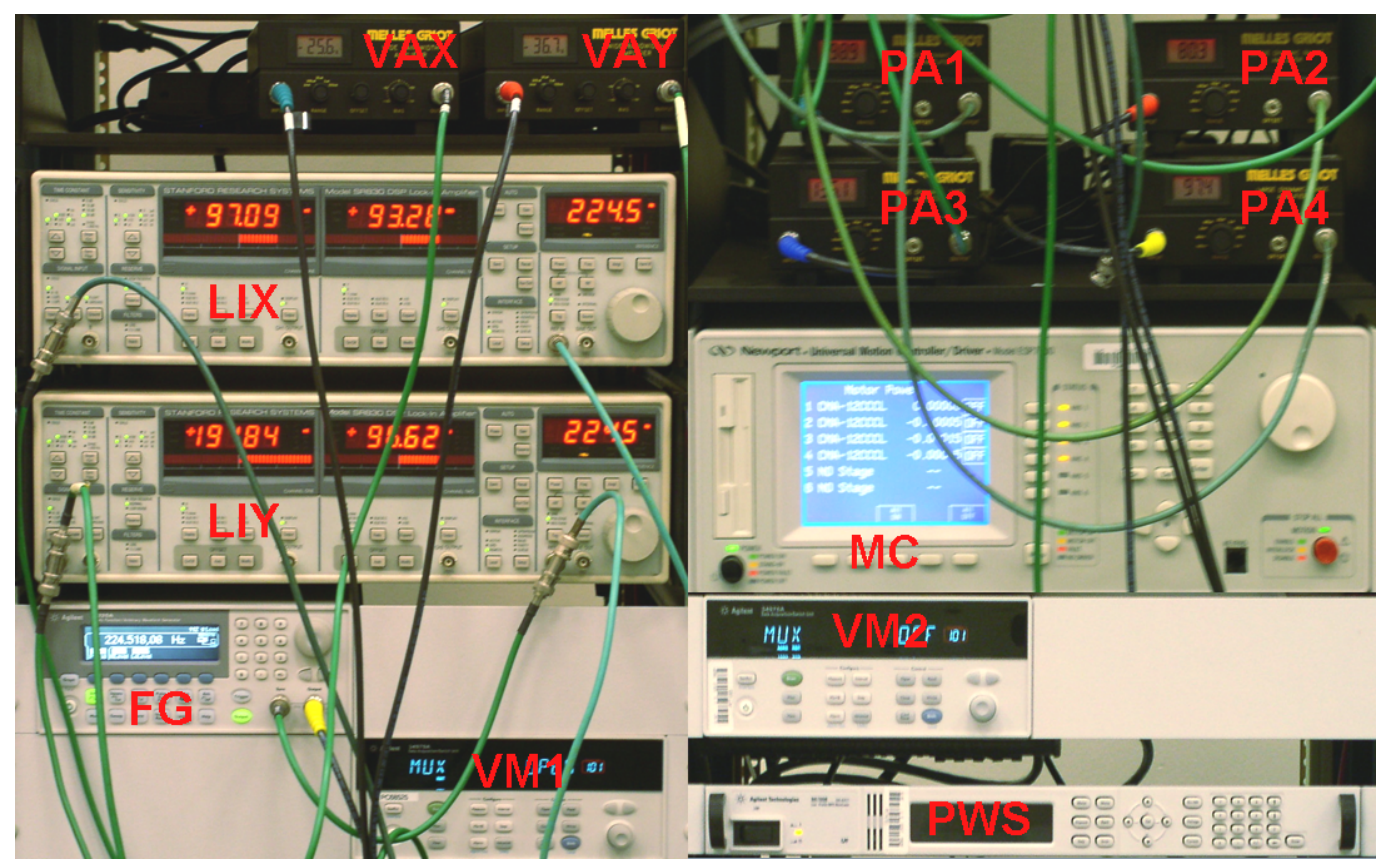

Figure 2: Electronics Setup

\subsection{Coordinate Measuring Machine}

We used a Leitz 12106 PMM (referred to as the CMM) to measure all tooling balls and pins.

The machine is located in the SLAC MMF facility, which is temperature controlled to within $0.1^{\circ} \mathrm{C}$. This allowed the machine to be calibrated to a repeatability of $0.2 \mu \mathrm{m}$ and an accuracy of $1.5 \mu \mathrm{m}$ over the whole range of $1 \mathrm{~m}$.

The CMM was controlled with software written in the Quindos7 environment.

We used two different probe setups. The first setup had short probes, while the second used probe extensions. The second setup was necessary, because the machine didn't have enough range to measure the pins. Because of the probe extensions involved in the setup we expect slightly larger errors in measuring the pin positions. However, these errors should be smaller than the uncertainty in the wire diameter or imperfections in the pin surfaces. Figure 1 shows the second CMM probe setup (PR) lying on the optical table. 
The diameters of all tooling balls on the position detectors and the magnet were carefully measured by the CMM. For all tests, we forced the software to fit its tooling ball measurements to these diameters, increasing the tests' accuracy and repeatability.

\subsection{Roller Cam Positioners}

The cam positioner system was built based on the design described in an LCLS technical note ([5]). We measured the geometry of the system and inserted the appropriate parameters into equations given in the note. The equations allowed us to convert the cam angles to the quadrupole center coordinates and back. However, because we don't know the parameters exactly, the conversions contain errors. This increases the number of iterations required to position the magnet on the wire.

Each cam positioner motor is controlled by a Parker E-series motor driver; all of the drivers are run by a Parker $6 \mathrm{~K}$ series motor controller.

\section{Measurements and Analysis}

\subsection{Repeatability of Position Detector and Magnet Align- ments}

We aligned the 4 position detectors and the magnet on the wire and used the CMM to measure the locations of their tooling balls. Two tooling balls were measured on each position detector, mimicking the way fiducialization will be performed. Three tooling balls were measured on the magnet, allowing us to check that all the relevant degrees of freedom remain constant. We repeated this procedure multiple times and measured the distribution of all tooling ball positions.

For the position detectors we calculated the variations in position distributions for the $x, y$, and $z$ coordinates independently and added those variations in quadrature. This number should give an accurate measure of how much each of the tooling balls tends to move around in three dimensions, giving the repeatability of the position detectors. The position detectors measure the wire position for only one degree of freedom, but we wanted to make sure that the tooling balls were not moving in any direction between measurements. 
The table below lists the repeatability (root of the sum of deviations squared) of the detector tooling ball positions.

\begin{tabular}{|l|r|r|}
\hline Detector & Ball 1 $(\mu m)$ & Ball 2 $(\mu m)$ \\
\hline PX1 & 1.3 & 1.4 \\
PY1 & 1.1 & 1.0 \\
PY2 & 2.2 & 2.1 \\
PX2 & 2.6 & 3.5 \\
\hline
\end{tabular}

For the magnet, we also calculated the variation for each direction. The table below lists the variation in the 3 tooling ball positions of the magnet for the relevant $x$ and $y$ directions.

\begin{tabular}{|l|r|r|r|}
\hline & Ball 1 $(\mu m)$ & Ball 2 $(\mu m)$ & Ball 3 $(\mu m)$ \\
\hline$x$ & 2.1 & 2.0 & 2.7 \\
$y$ & 1.8 & 2.1 & 1.7 \\
\hline
\end{tabular}

Note that these tests measure the repeatability of both the alignment and the CMM measurement. This mimics the actual fiducialization procedure.

\subsection{Position detector calibration}

The procedure for calibrating the position detectors was previously described in [4]. For this note we used an identical procedure, except instead of relying on indicator readings we used the CMM to determine the change in tooling ball positions. Knowing the change in position between flips and the distance between the two tooling balls allows us to calculate $X_{w}$ for each detector. $X_{w}$ is the distance from one of the tooling balls to the laser along the axis of the detector.

To make sure that imperfections in the detectors do not affect the calibration or measurements, we shimmed each detector so that its axis was perpendicular to the wire to within 2 mrads or less. We reduced the roll of all detectors with respect to gravity to $2 \mathrm{mrads}$ or less.

During fiducialization, we can measure the position of both tooling balls and re-establish the detector axis. We then offset the location of one of the tooling balls by $X_{w}$, locating the wire in space.

The table below lists the results of these measurements. 


\begin{tabular}{|l|r|r|}
\hline Detector & $D(\mathrm{~mm})$ & $X_{w}(\mathrm{~mm})$ \\
\hline PX1 & $94.814 \pm 0.001$ & $47.348 \pm 0.001$ \\
PY1 & $95.024 \pm 0.001$ & $47.520 \pm 0.002$ \\
PY2 & $95.126 \pm 0.004$ & $47.449 \pm 0.002$ \\
PX2 & $94.899 \pm 0.007$ & $47.442 \pm 0.005$ \\
\hline
\end{tabular}

All values in the table are in millimeters. ' $D$ ' is the distance between the two tooling balls on a detector. Each reported distance is the average of 2 distance measurements obtained by a slightly different procedure. The reported uncertainty in the distance value is based on the discrepancy of the two distance measurements. The next column lists the calibration constant averaged over many calibrations, followed by the uncertainty. The reported uncertainty is the error of the mean of multiple calibrations combined with the uncertainty of the distance value.

Measuring both tooling balls during calibration allowed us to introduce an extra check, by making sure that we get the same distance between the tooling balls before and after the flip.

\subsection{Position detector check}

The previous study of the vibrating wire system ([4]) relied on a FARO arm for position measurements. The FARO arm measures tooling balls with an accuracy of at most $25 \mu \mathrm{m}$. This limits its use to only checking for the presence of large errors in the system. In particular, the FARO arm was used to measure the position of the crossed pins on which the wire sits. From these pins, the wire location was determined and compared to the wire location calculated from position detectors. The procedure had to be done in multiple steps, because the range of the arm was not large enough to span the whole stand. This resulted in accumulation of error, so there was poor agreement between the detector and the crossed pin coordinates, with up to $100 \mu \mathrm{m}$ of difference.

We used the CMM to perform a similar check.

As mentioned, the wire position is determined by two crossed pins at each end of the wire. We used the CMM to measure the pin positions to within a few microns of accuracy. Assuming the wire diameter of $100 \pm 4 \mu \mathrm{m}$ the location of wire endpoints was calculated. The pins typically had a form of $2 \mu \mathrm{m}$. A few microns of error might have accumulated from the large measurement range and from using long probe extensions. Combining all 
the possible sources of error, the uncertainty in the endpoint locations is about $5 \mu m$.

The position detectors gave $2 x$ and $2 y$ coordinates at different points on the wire. From the wire position at the endpoints we calculated the expected coordinates of the wire at the detectors.

The results are given in the table below.

\begin{tabular}{|l|r|r|r|}
\hline Detector & Position $(\mathrm{mm})$ & Nominal $(\mathrm{mm})$ & Difference $(\mu \mathrm{m})$ \\
\hline PX1 & $333.072 \pm 0.003$ & $333.067 \pm 0.004$ & $4 \pm 7$ \\
PY1 & $262.767 \pm 0.002$ & $262.759 \pm 0.004$ & $8 \pm 6$ \\
PY2 & $262.745 \pm 0.005$ & $262.748 \pm 0.004$ & $-3 \pm 9$ \\
PX2 & $333.107 \pm 0.006$ & $333.089 \pm 0.004$ & $19 \pm 10$ \\
\hline
\end{tabular}

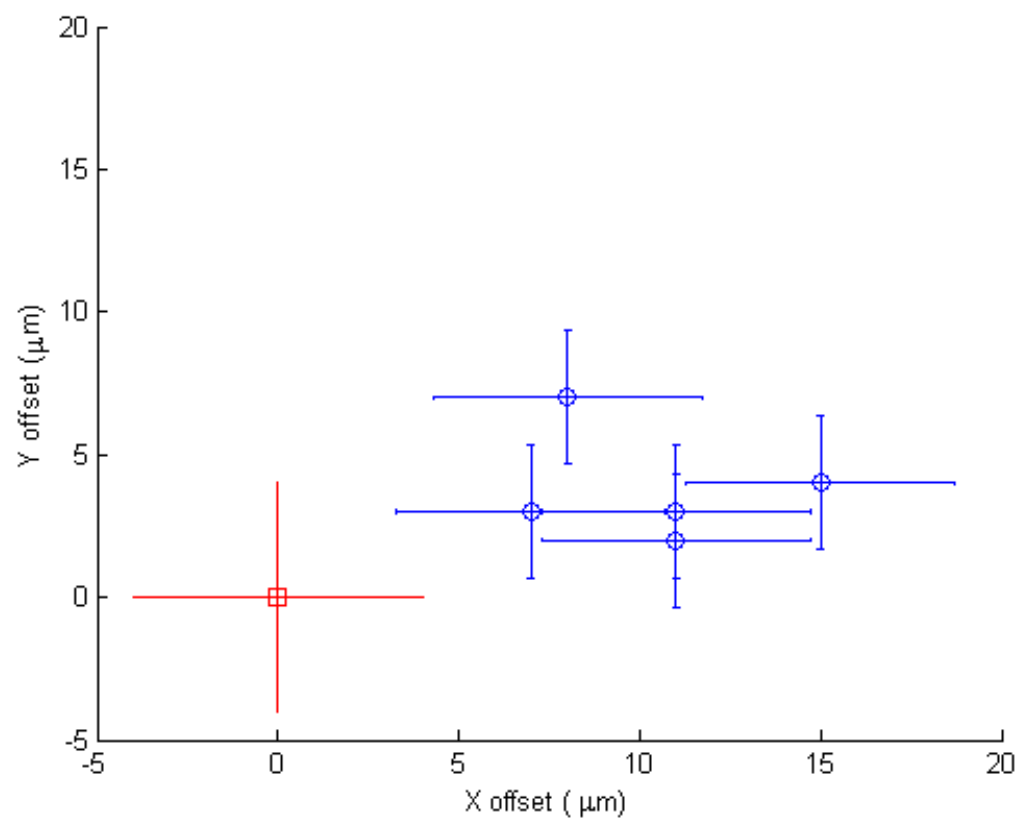

Figure 3: Position Detector Check

The red square is the position predicted from measuring the support pins at wire ends. The blue circles are the positions calculated from the wire detectors.

The rows PX1 and PX2 list the $x$ coordinate of the wire, PY1 and PY2 list the $y$ coordinates. The position calculated from each detector was averaged 
over 5 runs. We calculated its uncertainty by combining the error of the mean and the uncertainty of the calibration. The nominal position is the position calculated from wire ends. The last column is the difference between the average and the nominal positions.

We used the position detector calibrations to predict the wire coordinates at the quadrupole center. We then compared it to the coordinates we get by calculating from wire endpoints. The difference of the two is shown in Figure 3 as 5 blue circles, one circle per measurement set. The red square is at zero, representing where the circles would be if the position detectors were in complete agreement with the endpoint values. The error bars represent the combined uncertainties from both calibration and measurements. The average offset is $11 \pm 6 \mu m$ for $x$ and $4 \pm 6 \mu m$ for $y$.

Comparing the calculated quadrupole centers gives a lower offset, because the errors of both detectors are averaged. The offsets are also more meaningful in this case, because the procedure mimics fiducialization.

\subsection{Magnet Flip Test}

The quadrupole magnetic center is found by moving the wire until its vibration at the second resonance frequency goes to 0 .

The measured magnetic field consists of the field produced by the magnet and any external fields: $\vec{B}=\vec{B}_{m}+\vec{B}_{\text {ext }}$.

$$
\int_{0}^{L} \vec{B}_{m}(z) \sin \left(\frac{2 \pi z}{L}\right)=-\int_{0}^{L} \vec{B}_{e x t}(z) \sin \left(\frac{2 \pi z}{L}\right)
$$

If $\vec{B}_{\text {ext }} \neq 0$, it is possible for equation 1 to be satisfied when $\vec{B}_{m} \neq 0$ and the wire is not at the magnet center.

A uniform field, such as the Earth's, should not be a problem, since its second Fourier component is zero. However, the field may not be perfectly uniform around the setup and there may be other fields present. This may show up as an offset when the magnet is centered on the wire. To check the effect of external fields on the magnet, we did a magnet flip test for both $\mathrm{X}$ and Y directions. The test procedure is explained in [4].

\subsubsection{Measurements}

We aligned the quadrupole on the wire and measured its position with the CMM. We then flipped the magnet around the horizontal $(x)$ axis, aligned 
and measured its position again. Half of the difference in the two $x$ positions is the error in $x$ alignment, $k_{x}$, due to external fields. The table below lists the results of multiple measurements and their average.

\begin{tabular}{|l|r|}
\hline Point \# & $k_{x}(\mu m)$ \\
\hline 1 & $0.1 \pm 1$ \\
2 & $-2.3 \pm 1$ \\
3 & $1.4 \pm 1$ \\
\hline Average & $0.1 \pm 1$ \\
Variance & 2 \\
\hline
\end{tabular}

The uncertainty of the $k_{x}$ values was calculated from magnet $x$ alignment repeatability, determined to be $2 \mu m$ in a previous section.

\subsubsection{Y Measurements}

The same procedure was repeated for the $y$ direction, except the magnet was flipped around the $y$ axis. Half of the difference in the two $y$ positions is $k_{y}$, the error in $y$ alignment due to external fields. The table below lists the results of multiple measurements and their average.

\begin{tabular}{|l|r|}
\hline Point \# & $k_{y}(\mu m)$ \\
\hline 1 & $-1.1 \pm 1$ \\
2 & $0.9 \pm 1$ \\
\hline Average & $-0.1 \pm 2$ \\
Variance & 2 \\
\hline
\end{tabular}

\section{Discussion}

\subsection{Finding the Magnetic Center}

The magnet aligns on the wire accurately. The measured displacement in the $x$ direction is $0.1 \pm 1 \mu \mathrm{m}$. It is less than one micron and within the uncertainty of the measurement. The displacement in $y$ is also small at $-0.1 \pm 2 \mu m$, with the value less than its uncertainty.

Both of the offsets are smaller than the uncertainties, which suggests that the uncertainties were overestimated. Low sample size is a problem, so more flip tests need to be done. 
However, even with many more tests it is highly unlikely that the average offset will exceed one or two microns. This is a small part of the $25 \mu \mathrm{m}$ requirement on the fiducialization.

\subsection{Finding the Wire}

We calibrated all 4 detectors and checked their performance by looking at the wire ends. Detector PX2 had the worst results, in terms of repeatability, calibration uncertainty and in the detector check. The detector differed by up to $18 \mu \mathrm{m}$ from the wire position estimated from the pins.

However, when combining the data from both detectors to find the magnet coordinates the discrepancies reduce. For the $x$ coordinate the average offset was $11 \mu \mathrm{m}$ with a standard deviation of $6 \mu \mathrm{m}$. For the $y$ coordinate it was even smaller, at $4 \mu \mathrm{m}$ with a standard deviation of $6 \mu \mathrm{m}$.

The fact that the offset is not 0 means that there might be a problem with detector calibrations. Detector PX2 is particularly suspect, because it performed the worst for all the measurements.

There was high uncertainty in the detector check. Most of it comes from uncertainty in the wire diameter.

During the magnet flip test the wire seemed to displace significantly from run to run. The magnet displaced with the wire, so that the relative shift of the wire to the magnet remained small. Actually, the same data was used for both the magnet flip and the detector check tests. This confirms that the detectors reliably find the wire. It also suggests that the distribution of position between the runs, as observed in the detector check, is not because of poor detector alignment, but because the wire center actually shifted.

\subsection{Measuring Tooling Balls}

The fiducialization procedure requires tooling balls on both the magnet and the position detectors to be accurately measured. The CMM used in these tests is calibrated to better than $1 \mu \mathrm{m}$ of accuracy in the relevant range. Most of the tests in this note rely on this accuracy. While using the CMM we observed a repeatability of better than $1 \mu \mathrm{m}$ on all measurements of static objects. Therefore, we believe that the CMM can easily find the relative position of tooling balls to an accuracy of better than $2 \mu m$, which is well within the fiducialization requirements. 


\section{Component Performance Analysis}

Before the vibrating wire system can be used for LCLS, it is important to make sure that the system is thoroughly understood and all of its components behave as expected.

Wire resonance frequency, wire sag, vibration detectors, position detectors, decay constant, sensitivity, and movement coupling were previously studied [4]. Most of the analysis applies to the setup in this note. However, parts of the system have changed and we did additional checks to make sure that we still understand the system.

\subsection{Cam System Positioning Test}

We did a test to make sure that the cam system behaves as expected. The cam positioners calculate the quadrupole center based on potentiometer readings at each cam. The center can be calculated incorrectly for many reasons. These include bad formulas, bad parameters for the formulas, incorrect potentiometer zero positions as well as imperfections in the machining and other defects.

To measure the actual behavior of the cam positioner system we attached 5 indicators to the quadrupole support stand. They allowed us to indirectly measure movement in 5 degrees of freedom: X, Y, Roll, Pitch, and Yaw. However, because we didn't position the indicators perfectly, there were errors introduced in the conversion from indicator readings to the support coordinates.

We did 4 separate tests, one for each of X, Y, Pitch and Yaw directions. The cam positioners moved in steps along each direction. We recorded the position readings from both the cam positioners and the indicators.

The results are summarized in Figures 4 through 7, one for each direction of motion. The data plotted in the figures is the difference between the indicator data and the positions calculated from cam potentiometers. For each test and coordinate, we offset the data by a constant value to make the average go to zero; the absolute difference between the indicator and cam potentiometer readings is not meaningful.

On all 4 plots, the $x$ and $y$ positions are plotted in microns. Each unit of pitch, roll or yaw represents a $10 \mu \mathrm{rad}$ angle. 


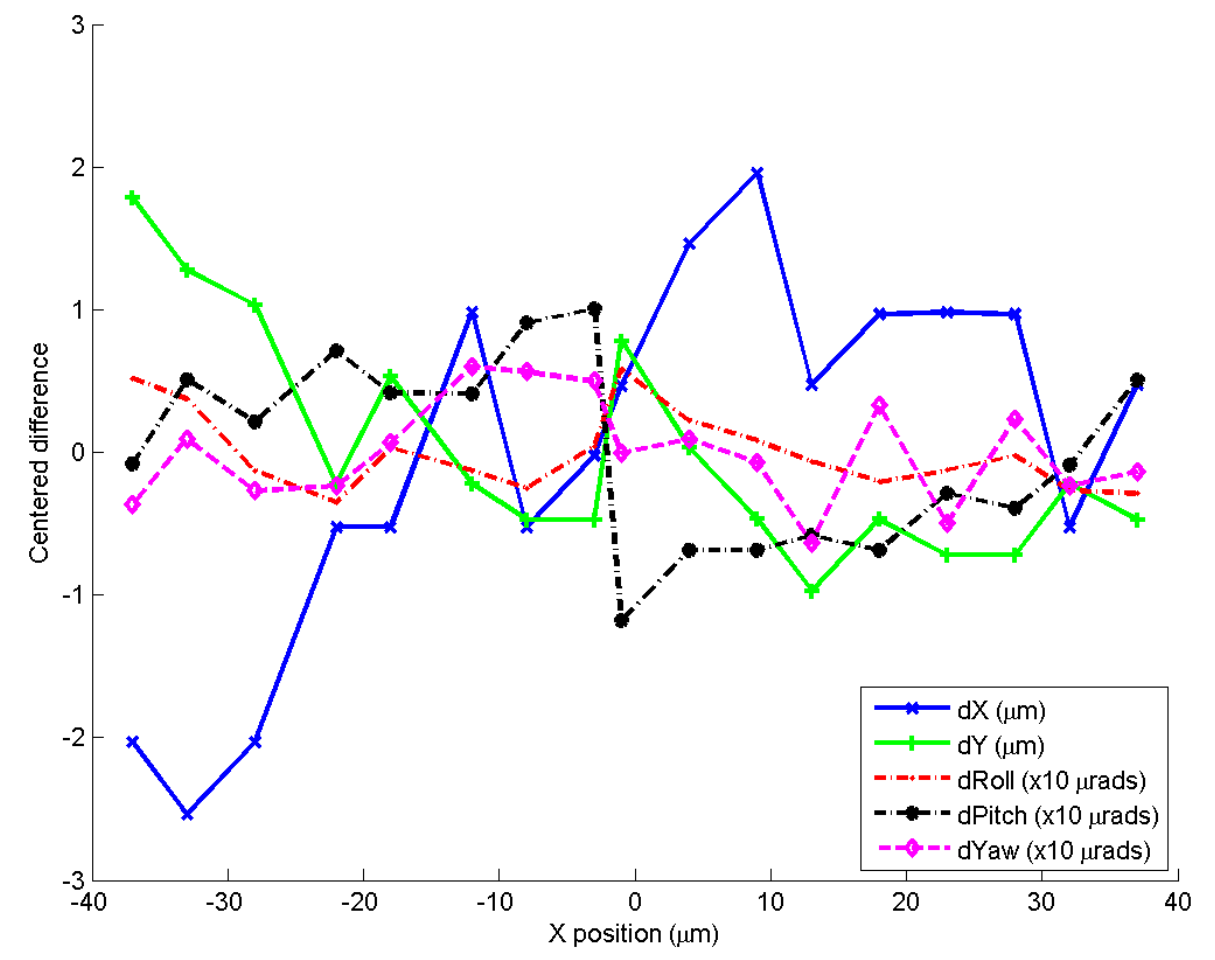

Figure 4: Movement in X

\subsubsection{X movement}

In Figure 4 we told the cam positioners to move in the $x$ direction in a range of approximately $80 \mu \mathrm{m}$, while keeping the other parameters constant. The difference between the potentiometer and indicator readings was plotted.

The difference in all three angles (roll, pitch, yaw) stays within $\pm 12 \mu \mathrm{rads}$ for all points. The difference in $y$ measurements stays between $-1 \mu m$ and $2 \mu m$, while the difference in $x$ has a larger range between $-2.5 \mu m$ and $2 \mu m$.

The differences come both from the imperfections in the cam positioners and errors in indicator readings. From this plot we conclude that when the $x$ coordinate is moved within a $80 \mu m$ range, all of the angle readings are correct to within $12 \mu \mathrm{rads}$ or better.

We believe that the discrepancies for both $x$ and $y$ mostly come from the 
indicator errors. The surfaces on which the indicators were placed were not perfectly smooth. In addition, we assumed that the indicators were placed perpendicular to the surfaces. However, we positioned them by eye, so they were likely off by a few degrees, introducing cosine errors into the calculations.

The results show that the errors in $x$ and $y$ do not exceed a few microns. We expect the errors to drop significantly if we could remove the indicator setup errors from the calculations.

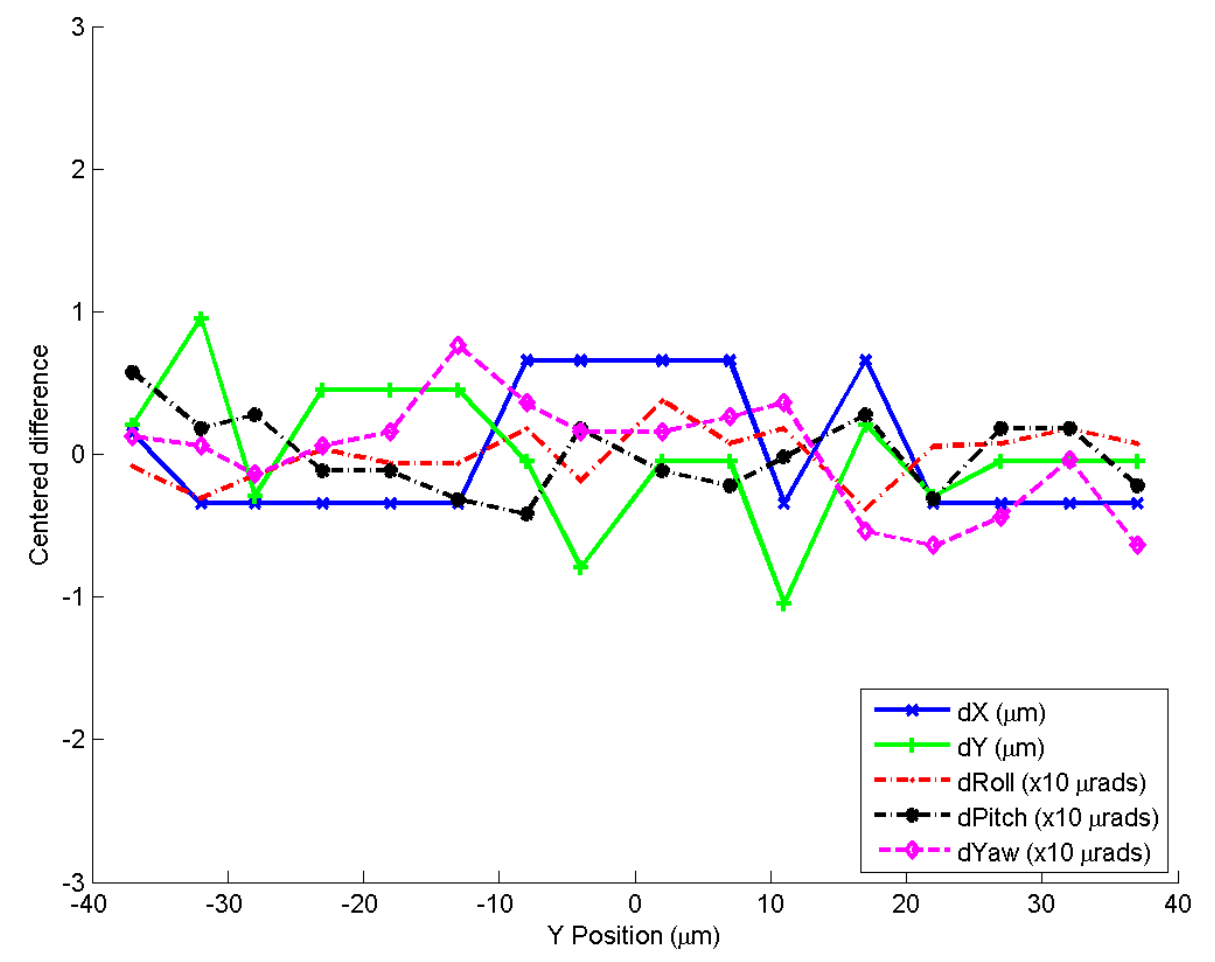

Figure 5: Movement in $\mathrm{Y}$

\subsubsection{Y movement}

We instructed the cam positioners to move in the $y$ direction in a range of approximately $80 \mu \mathrm{m}$. The resulting differences in readings are plotted in Figure 5 . 
The results are similar to the ones for movement in $x$, with smaller discrepancies for all of the degrees of freedom. The angle differences stayed within $\pm 7 \mu$ rads. The $x$ and $y$ errors were always within $\pm 1 \mu \mathrm{m}$.

When the support platform is moved by small amounts in $y$, we expect the positioning errors to be within these values.

\subsubsection{Pitch movement}

We instructed the cam positioners to move the platform in pitch, while keeping all of the other angles and the $x$ and $y$ positions of the quadrupole center constant. The results are plotted in Figure 6.

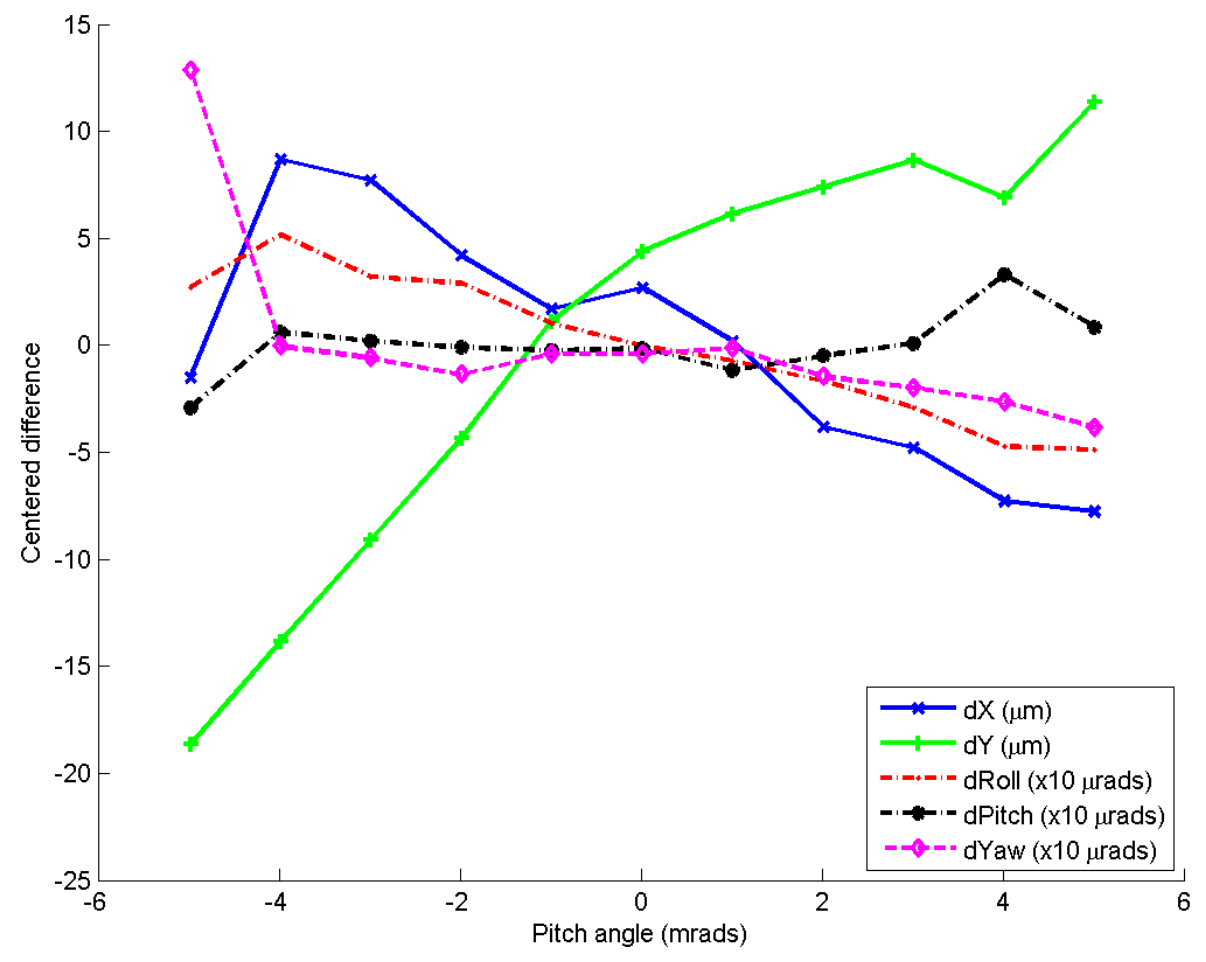

Figure 6: Movement in Pitch

The angles stay within $\pm 50 \mu \mathrm{rads}$ for most of the time. The exception is the first point in yaw, which goes up to $130 \mu$ rads for an unknown reason. It 
is possible that this is because of uneven surface under one of the indicators, resulting in a sudden jump when the surface is moved by a large amount laterally. Notice that the difference in $x$ position also jumps by more than $10 \mu \mathrm{m}$ after the first point. The indicators involved in measuring $x$ were also used for yaw calculations, but not for $y$, roll or pitch. So, an error in a indicator reading would be consistent with the observed results.

The $y$ reading seems to depend on pitch, according to the plot. This is to be expected, because of the way $y$ is calculated. The measured distances from the center of the quadrupole to the indicators are not perfectly accurate. The cam positioners attempt to keep the quadrupole center steady, but this is not the same point that the indicators use, so there is a change in $y$ with a change in pitch. This mimics the quadrupole fiducialization process, in which the quad center will not be at the point used by cam positioners.

The $x$ value also seems to depend on pitch, probably because of imperfect positioning of indicators.

\subsubsection{Yaw movement}

As seen from Figure 7, moving the magnet in yaw results in large discrepancies for some of the coordinates.

Pitch and roll both have errors similar to the previous tests, never exceeding $\pm 8 \mu \mathrm{rads}$. The $y$ error is worse than the $x$ and $y$ movements tests with the range of $\pm 3 \mu \mathrm{m}$.

The yaw difference shows an even bigger jump than in the pitch movement test, changing by almost $250 \mu \mathrm{rads}$ in the first step. It continues to change by smaller values throughout the rest of the test.

The $x$ position seems to depend on yaw. The coupling is expected, because of the way $x$ position is calculated from indicator readings. Just like

for $y$, the distances from the quadrupole center to the indicators are not perfectly known. This results in errors when trying to separate the movement into individual components. Even larger coupling will likely exist during the fiducialization process, because the support stand center will not exactly coincide with the quadrupole center.

Also, the stand might not be best suited for yaw adjustments. In the design of the system the distance between the back and front cam positioners was assumed to be large. However, in this case it is not that large, so it amplifies any imperfections in the cams or the support that sit on the cams. 


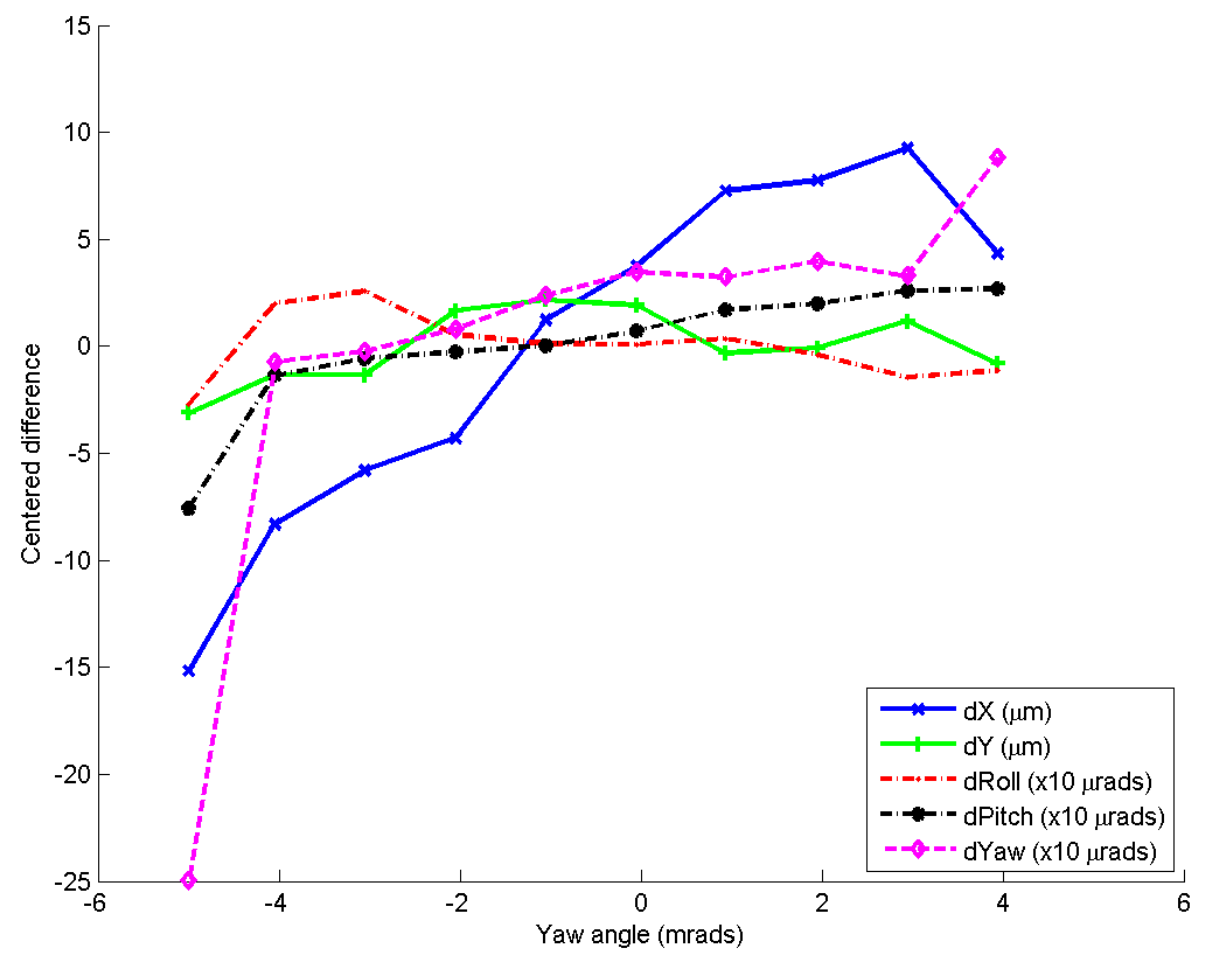

Figure 7: Movement in Yaw

\subsection{Wire Sag}

The force of gravity produces a known sag in the wire that can be calculated precisely from the fundamental frequency [2]. Taking the fundamental frequency from the measurements as $115 \mathrm{~Hz}$, the maximum sag was approximated to be $23 \mu m$.

We calculated the sag at each point by assuming that the wire takes on the shape of a catenary. The sag magnitudes at all position detectors and the magnet are listed in the table below. 


\begin{tabular}{|l|l|}
\hline Part & Sag $(\mu m)$ \\
\hline PX1 & 7 \\
PY1 & 10 \\
PX2 & 22 \\
PY2 & 23 \\
\hline Magnet & 17 \\
\hline
\end{tabular}

These results were used to adjust the wire values for the position detector check earlier in the text.

\subsection{Wire Diameter}

The manufacturer reports the wire diameter as $100 \mu m$. We optically measured the diameter of two different pieces of the wire. The first piece of wire was not previously used. The second piece sat for 10 days under a tension of $5.9 \mathrm{~N}$, the same tension the wire would experience during quadrupole fiducialization. Four points were measured on each wire.

The unused wire had a diameter of $110 \pm 1 \mu \mathrm{m}$. The second wire had a diameter of $103 \pm 1 \mu \mathrm{m}$. The diameter of the second wire is significantly smaller, suggesting that the wire stretched when it was tensioned.

The wire will have a slightly smaller diameter when it is under tension. For the wire position detector check, we used the nominal diameter of $100 \mu \mathrm{m}$ to calculate the offset of wire center from the pins. The uncertainty of the diameter was assumed to be $4 \mu m$.

\subsection{Magnet Alignment Procedure}

The software aligns the magnet on the wire by separately adjusting pitch, yaw, $x$, and $y$ to make them give the smallest wire vibration amplitude. If in order to do that the magnet had to be moved significantly, the program realigns all directions. The process repeats until the magnet stops moving between iterations. At that point, the magnet is assumed to be aligned on the wire.

For the magnet flip tests in this note, the program was set to align the magnet to within $1 \mu \mathrm{m}$ in both $x$ and $y$ directions, and to within $0.1 \mathrm{mrad}$ and $0.3 \mathrm{mrad}$ in pitch and yaw, respectively.

Pitch and yaw had a much larger noise to signal ratio and were more difficult to align, while $x$ and $y$ showed good sensitivity to movements as small as $1 \mu m$. 
The cam positioners are physically capable of moving the platform in hundreds of microns in $x$ and $y$ and by more than $5 \mathrm{mrad}$ of pitch and yaw.

We found that the program is capable of aligning the magnet when it is offset by $100 \mu \mathrm{m}$ in both $x$ and $y$ and by 2 mrads of both pitch and yaw. However, it takes much longer for the program to do so and it is better to use the manual adjustment on the support stand to roughly align the magnet by hand.

\section{Conclusion}

The first step of fiducialization is aligning the magnet on the wire. In this note we found that the repeatability of alignment is $2 \mu \mathrm{m}$ with an offset of no more than $2 \mu m$.

The second step, finding the wire, had an error of no more than $11 \pm 6 \mu \mathrm{m}$ for $x$ and $4 \pm 6 \mu m$ for $y$. In addition, the repeatability in the detector alignment was $2 \mu m$ on average.

The third step, of measuring and comparing the position of tooling balls, has an error of no more than $2 \mu \mathrm{m}$ because of the high accuracy of the CMM.

It is safe to assume that all of the errors are independent, so that they can be added in quadrature. For $x$ direction adding $2 \mu m, 2 \mu m, 11 \mu m, 6 \mu m$, $2 \mu m$, and $2 \mu m$ in quadrature gives $13 \mu m$. For $y$ direction a similar addition gives $8 \mu m$.

This means that a single quadrupole fiducialization will give a coordinate that is offset from the actual magnetic axis by no more than $13 \mu \mathrm{m}$ in $x$ direction and $8 \mu \mathrm{m}$ in $y$ direction. This is well within the fiducialization requirement of $25 \mu \mathrm{m}$ for each direction.

Even a direct addition of all errors gives only $25 \mu \mathrm{m}$ for $x$ and $18 \mu \mathrm{m}$ for $y$. But the case that all possible errors will combine in the same way is extremely unlikely, so it is safe to assume that the fiducialization offset will stay well within the requirements.

There can be improvements made to the system. For example, one can investigate the large disagreement in the wire check for the 4 th position detector. If there is a calibration error, fixing it might improve the accuracy. However, even without any adjustments, the system studied in this note is sufficiently accurate to fiducialize the LCLS quadrupoles. 


\section{References}

[1] H. D. Nuhn et al. General undulator system requirements. LCLS Physics Requirements Document 1.4-001, SLAC, 2005.

[2] Z. Wolf. A vibrating wire system for quadrupole fiducialization. LCLS Technical Note LCLS-TN-05-11, SLAC, 2005.

[3] Z. Wolf et al. Lcls undulator quadrupole fiducialization plan. LCLS Technical Note LCLS-TN-07-7, SLAC, 2007.

[4] M. Levashov and Z. Wolf. Set up and test results for a vibrating wire system for quadrupole fiducialization. LCLS Technical Note LCLS-TN06-14, SLAC, 2006.

[5] G. Bowden. Roller cam positioners. LCLS Technical Note LCLS-TN-0528, SLAC, 2005. 